\section{The Burden of Infectious Gastroenteritis in Elderly Residents and Staff of Long-Term Care Facilities, Australia}

\author{
M. D. Kirk, BAppSci, MAppEpid; \\ C. R. M. Moffatt, BBusHealthAdmin, MPH, MAppEpid; \\ G. V. Hall, BSc(Med), MBBS, PhD; N. Becker, MSc, PhD; \\ R. Booy, MBBS, MSc, MD, FRACP, FRCPCH; \\ L. Heron, MBChB, FRCPA, FAFPHM; \\ R. MacIntyre, MBBS, MAppEpid, PhD, FRACP, FAFPMH; \\ D. E. Dwyer, MBBS, MD, FRACP, FRCPA; \\ R. Lindley, MBBS, MD, FRCP(Edin), FRACP
}

We estimated the incidence of gastroenteritis in 16 Australian longterm care facilities. During 12 months' surveillance, 245 (96\%) of 254 episodes of gastroenteritis among long-term care residents were associated with 17 outbreaks in 11 facilities. Incidence in long-term care residents was 0.64 episodes per 1,000 bed-days ( $95 \%$ confidence interval, 0.29-1.42).

Infect Control Hosp Epidemiol 2010; 31(8):860-863

In Australia during 2007, outbreaks of gastroenteritis affecting long-term care facility (LTCF) residents accounted for 1,010 (54\%) of 1,882 gastroenteritis outbreaks reported nationwide. ${ }^{1}$ LTCFs may experience gastroenteritis outbreaks caused by such infectious agents as Clostridium difficile, Clostridium perfringens, noroviruses, rotaviruses, and Salmonella., ${ }^{2,3}$ Despite the frequency of outbreaks, little is known about the incidence of gastroenteritis in LTCFs. Internationally, the burden of gastroenteritis in LTCF residents is $0.04-1.9$ episodes per 1,000 resident-days. ${ }^{4,5}$ The variation in incidence in published studies is probably due to differing definitions of gastroenteritis and varying intensity of surveillance. To assess the burden of gastroenteritis in LTCFs, we studied residents and staff of 16 facilities owned by a single company in New South Wales, Australia, during the period from October 25, 2007, through October 31, 2008.

METHODS

Study nurses made weekly telephone calls to directors of nursing or facility managers to ascertain cases of gastroenteritis among residents and staff. LTCF staff members recorded the onset and cessation of illness, symptoms, and outcomes. An episode of gastroenteritis was defined as at least 3 loose stools that were different from normal bowel habits during a 24hour period, was considered infectious, and was separated from another episode of gastroenteritis by at least 48 hours.

To calculate incidence, we divided the number of episodes of gastroenteritis in long-term care (LTC) residents and assisted living area (ALA) residents (ALA residents required only partial assistance with activities of daily living) in each facility by the number of occupied bed-days. We estimated the mean incidence for all facilities and calculated 95\% confidence intervals (CIs) assuming a negative binomial distribution and using robust variance estimation to account for possible correlation in the data according to LTCF.

We defined an outbreak as at least 2 persons in a facility becoming ill within 72 hours of each other. The etiology of outbreaks was attributed to a specific pathogen when at least 1 fecal test was positive for a recognized pathogen. Episodes that were not part of defined outbreaks were considered sporadic.

Data analysis was performed using Stata, version 10.1 (StataCorp). Institutional ethics committees approved the study, and there was no attempt to influence treatment or testing practices of physicians. Facilities experiencing outbreaks of gastroenteritis were reminded of their legal obligations to notify public health authorities.

\section{RES ULTS}

During the 12 months of surveillance, there were a total of 567,894 bed-days under surveillance (Table 1), including 125,156 bed-days in ALAs. In total, 346 (84\%) of 411 reported episodes met the case definition. Two hundred fifty-four episodes occurred among LTC residents living in 12 (75\%) of 16 facilities, compared with 22 episodes reported among ALA residents living in 2 (29\%) of 7 facilities (Table 1). The mean age of LTC resident case patients was 84.5 (95\% CI, 83.2$85.7)$, and $176(69 \%)$ of 254 were women. The mean age of ALA resident case patients was 84.8 (95\% CI, 83.1-86.6), and $15(68 \%)$ of 22 were women.

The mean facility incidence of gastroenteritis episodes in LTC residents during surveillance was 0.64 episodes per 1,000 bed-days (95\% CI, 0.29-1.42). Among ALA residents, the mean facility incidence was 0.15 episodes per 1,000 bed-days (95\% CI, 0.03-0.77). There were 70 episodes of gastroenteritis reported among staff, equating to a mean facility rate of 0.05 episodes of gastroenteritis per person per year.

During the surveillance period, there were 17 outbreaks in 11 different facilities. The median size of outbreaks was 5 persons (range, 2-72 persons), and 9 (53\%) of 17 outbreaks occurred in winter. During the surveillance period, 325 (94\%) of 346 episodes of gastroenteritis were associated with an outbreak (Figure 1), including 245 (96\%) of 254 episodes among LTC residents, 19 (86\%) of 22 episodes among ALA residents, and $61(87 \%)$ of 70 episodes among staff. Gastroenteritis episodes in LTC residents were more likely to be associated with an outbreak than were episodes in staff (relative risk, 1.1 [95\% CI, 1.0-1.2]), but there was no significant difference between LTC residents and ALA residents in the proportion of episodes that were associated with an outbreak 
тав в 1. Episodes of Gastroenteritis Meeting Case Definition in Long-Term Care (LTC) Residents, Assisted Living Area (ALA) Residents, and Staff of 16 Long-Term Care Facilities in New South Wales, Australia, 2007-2008

\begin{tabular}{|c|c|c|c|c|c|c|c|c|}
\hline \multirow[b]{2}{*}{ Facility } & \multicolumn{3}{|c|}{ LTC residents } & \multicolumn{3}{|c|}{ ALA residents } & \multirow[b]{2}{*}{$\begin{array}{c}\text { Staff } \\
\text { episodes }\end{array}$} & \multirow[b]{2}{*}{$\begin{array}{c}\text { Total } \\
\text { episodes }\end{array}$} \\
\hline & Episodes & Bed-days & $\begin{array}{l}\text { Incidence, } \\
\text { episodes per } \\
1,000 \text { bed-days }\end{array}$ & Episodes & Bed-days & $\begin{array}{l}\text { Incidence, } \\
\text { episodes per } \\
1,000 \text { bed-days }\end{array}$ & & \\
\hline A & 0 & 23,544 & 0 & $\ldots$ & $\ldots$ & $\ldots$ & 1 & 1 \\
\hline B & 68 & 34,838 & 2.0 & 0 & 22,703 & 0 & 39 & 107 \\
\hline $\mathrm{C}$ & 4 & 34,930 & 0.11 & $\ldots$ & $\ldots$ & $\ldots$ & 0 & 4 \\
\hline $\mathrm{D}$ & 2 & 31,393 & 0.06 & $\ldots$ & $\ldots$ & $\ldots$ & 0 & 2 \\
\hline $\mathrm{E}$ & 12 & 21,694 & 0.55 & $\ldots$ & $\ldots$ & $\ldots$ & 9 & 21 \\
\hline $\mathrm{F}$ & 90 & 24,220 & 3.7 & 18 & 20,117 & 0.89 & 0 & 108 \\
\hline G & 6 & 28,234 & 0.21 & $\ldots$ & $\ldots$ & $\ldots$ & 1 & 7 \\
\hline $\mathrm{H}$ & 0 & 29,374 & 0 & 0 & 12,373 & 0 & 1 & 1 \\
\hline I & 5 & 36,757 & 0.14 & 0 & 17,489 & 0 & 1 & 6 \\
\hline $\mathrm{J}$ & 0 & 29,310 & 0 & 4 & 22,052 & 0.18 & 1 & 5 \\
\hline K & 8 & 29,729 & 0.27 & $\ldots$ & $\ldots$ & $\ldots$ & 1 & 9 \\
\hline $\mathrm{L}$ & 5 & 25,304 & 0.20 & $\ldots$ & $\ldots$ & $\ldots$ & 1 & 6 \\
\hline M & 1 & 36,119 & 0.03 & $\ldots$ & $\ldots$ & $\ldots$ & 0 & 1 \\
\hline $\mathrm{N}$ & 21 & 11,729 & 1.8 & 0 & 17,744 & 0 & 6 & 27 \\
\hline $\mathrm{O}$ & 0 & 20,371 & 0 & 0 & 12,678 & 0 & 1 & 1 \\
\hline $\mathrm{P}$ & 32 & 25,192 & 1.3 & $\ldots$ & $\ldots$ & $\ldots$ & 8 & 40 \\
\hline Total & 254 & 442,738 & $\ldots^{a}$ & 22 & 125,156 & $\ldots^{a}$ & 70 & 346 \\
\hline
\end{tabular}

a Incidence is calculated for LTC residents and ALA residents in individual facilities only.

(relative risk, 1.1 [95\% CI, 0.9-1.3]). In total, 66 fecal specimens were collected during the surveillance period, all of which were obtained during outbreaks. The etiology was known for 8 (47\%) of 17 outbreaks: norovirus affected 185 people during 6 outbreaks, and C. perfringens affected 77 people during 2 outbreaks.

\section{I S C US S I O N}

Our study suggests that Australian LTCF residents can expect to experience gastroenteritis once every 5 years, which is less frequent than for people aged at least 65 years living in the community. ${ }^{6}$ Our mean estimate of gastroenteritis incidence was based on variation observed in 16 different facilities, some of which had higher incidence than surveillance studies reported in the literature. ${ }^{4,7}$ The high incidence in some facilities was due to the occurrence of multiple outbreaks during surveillance. This emphasizes the importance of training LTCF staff in infection control and outbreak management, because facilities may experience outbreaks every 1-2 years. ${ }^{8}$

In our study, sporadic gastroenteritis was rare, although our data collection may have been biased to detect outbreakassociated cases because of the requirement for facilities to notify authorities of outbreaks. However, our estimated incidence of gastroenteritis was comparable to those of other investigators, ${ }^{9}$ and it was unlikely that we missed large numbers of sporadic cases that would reduce the proportion of outbreak-associated disease. Supposing we had missed $80 \%$ of sporadic cases in study facilities, the rate of sporadic gas- troenteritis in residents would only be 0.1 episodes per 1,000 bed-days, which represents only a small proportion of the burden of infectious gastrointestinal illness. We used a sensitive definition for an outbreak that was commonly used in Australia. The use of this definition may have inflated the proportion of disease that was associated with an outbreak, although most outbreaks consisted of several epidemiologically related cases.

An important finding from this study was that not all outbreaks were due to norovirus. Two large outbreaks were confirmed as C. perfringens intoxication, and there were also outbreaks of unknown etiology that were suspected to be due to this pathogen. It was surprising that $C$. difficile was not detected during the study, although we did not ascertain whether stool samples were tested for this pathogen. During outbreaks, investigators should identify etiological agents causing gastroenteritis in LTCF residents, because different management strategies are required for different pathogens. ${ }^{1}$

We believe that cases in ALA residents and staff were less likely to be reported than cases in LTC residents, although we were unable to validate surveillance of these groups in the facilities. Our methods were similar to those of other published studies, ${ }^{9}$ and we do not believe that any systematic bias was introduced. If staff members were away from work for while ill and for 2 days after cessation of symptoms to minimize spread, it would equate to 372 days of work lost annually in these 16 facilities.

We used a simple definition of gastroenteritis used previously in community-based studies of gastroenteritis and studies in LTCFs. ${ }^{6,10}$ We excluded $65(16 \%)$ of 411 reported 


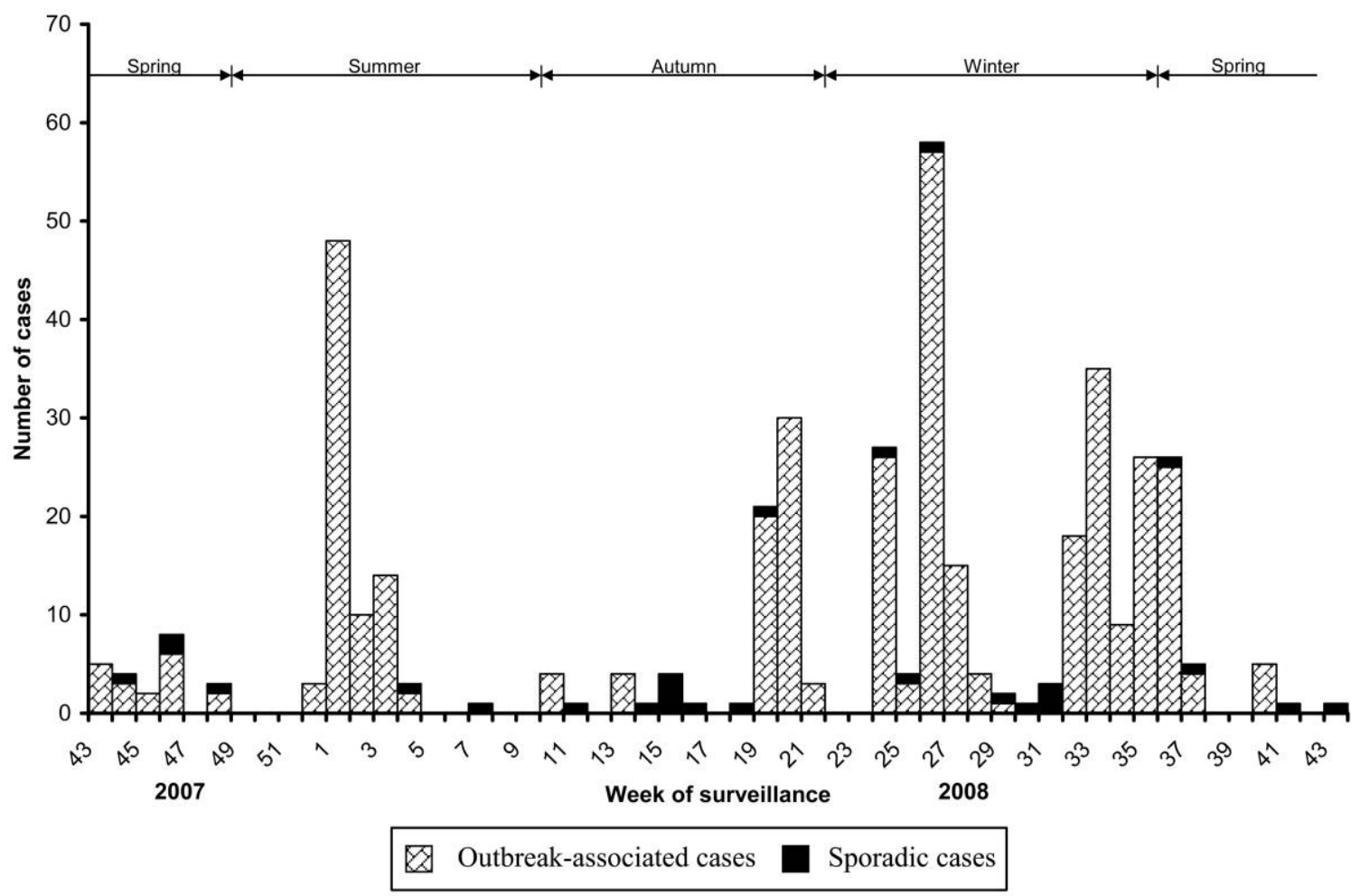

FIGURE 1. Sporadic and outbreak-associated cases of gastroenteritis in 16 Australian long-term care facilities, according to week of surveillance, during the period from October 25, 2007, through October 31, 2008.

cases, because they did not meet the case definition as a result of mild illness or lack of diarrhea. We may have inadvertently included some cases of disease due to $C$. perfringens that did not meet the case definition, because some episodes during these outbreaks were mild but details were not recorded (J. Musto, MPH, NSW Health, verbal communication, May 1, 2009). However, misclassification would not have a substantial effect on calculation of incidence, and cases truly represented outbreak-associated gastroenteritis.

This study highlighted the burden that outbreak-associated gastroenteritis places on LTCFs and residents. It is important to note that the study findings we report may be specific to Australia. Future surveillance studies of the incidence of gastroenteritis in LTCF residents should incorporate an extensive work-up of stool specimens that includes norovirus, C. perfringens, and C. difficile.

\section{ACKNOWLEDGMENTS}

We thank the long-term care provider, the managers of the individual facilities, and the research nurses for their assistance with this study.

Financial support. The Australia Department of Health and Ageing funded this study under the OzFoodNet program of work. M.D.K. is a National Health and Medical Research Council scholar. R.L. is supported by an infrastructure grant from NSW Health.

Potential conflicts of interest. All authors report no conflicts of interest relevant to this article.
From the National Centre for Epidemiology and Population Health, Australian National University (M.D.K., G.V.H., N.B.), OzFoodNet, Australian Government Department of Health and Ageing (M.D.K.), and OzFoodNet, Australian Capital Territory Health (C.R.M.M.), Canberra, the National Centre for Immunisation Research and Surveillance of Vaccine Preventable Diseases, Children's Hospital at Westmead (R.B., L.H., R.M.), Sydney Medical School, University of Sydney (R.B., L.H., D.E.D., R.L.), the School of Public Health and Community Medicine, University of New South Wales (R.M.), and the Centre for Infectious Diseases and Microbiology, Westmead Hospital (D.E.D.), Sydney, Australia.

Address correspondence to Martyn Kirk, BAppSci, MAppEpid, Australian National University, Canberra, ACT 0200, Australia (martyn.kirk@anu.edu.au).

Received December 14, 2009; accepted January 31, 2010; electronically published June 28, 2010.

(C) 2010 by The Society for Healthcare Epidemiology of America. All rights reserved. 0899-823X/2010/3108-0016\$15.00. DOI: 10.1086/654000

\section{REFERENCES}

1. Kirk M, Veitch M, Hall G. Food safety: gastroenteritis and foodborne disease in elderly people living in long-term care. Clin Infect Dis 2010;50: 397-404.

2. Laffan AM. Burden of Clostridium difficile-associated diarrhea in a longterm care facility. J Am Geriatr Soc 2006;54:1068-1073.

3. Ryan MJ, Wall PG, Adak GK, et al. Outbreaks of infectious intestinal disease in residential institutions in England and Wales 1992-1994. J Infect 1997;34:49-54.

4. Heudorf U, Schulte D. Surveillance of nosocomial infections in a long- 
term care facility: incidence and risk factors [in German]. Bundesgesundheitsblatt Gesundheitsforschung Gesundheitsschutz 2009;52:732-743.

5. Scheckler WE, Peterson PJ. Infections and infection control among residents of eight rural Wisconsin nursing homes. Arch Intern Med 1986;146: 1981-1984.

6. Scallan E, Majowicz SE, Hall G, et al. Prevalence of diarrhoea in the community in Australia, Canada, Ireland, and the United States. Int Epidemiol 2005;34:454-460.

7. Engelhart ST, Hanses-Derendorf L, Exner M, Kramer MH. Prospective surveillance for healthcare-associated infections in German nursing home residents. J Hosp Infect 2005;60:46-50.
8. Eastwood K, Osbourn M, Francis L, et al. Improving communicable disease outbreak preparedness in residential aged care facilities using an interventional interview strategy. Australas J Ageing 2008;27:143-149.

9. Stevenson KB, Moore J, Colwell H, Sleeper B. Standardized infection surveillance in long-term care: interfacility comparisons from a regional cohort of facilities. Infect Control Hosp Epidemiol 2005;26:231-238.

10. Nicolle LE, McIntyre M, Zacharias H, MacDonell JA. Twelve-month surveillance of infections in institutionalized elderly men. J Am Geriatr Soc 1984;32:513-519. 